\title{
Strong Effects of Subphysiological Temperature on the Function and Plasticity of Mammalian Presynaptic Terminals
}

\author{
Kristina D. Micheva and Stephen J. Smith \\ Department of Molecular and Cellular Physiology, Stanford University, Stanford, California 94305
}

\begin{abstract}
Most cellular processes are known to be strongly temperature dependent. Nevertheless, a large fraction of studies of mammalian synaptic function have been and are performed near room temperature (i.e., at least $10^{\circ} \mathrm{C}$ below physiological temperature). Here, we examined the effects of temperature on presynaptic function in primary cultures of rat hippocampal neurons. FM dyes, VAMP (vesicle-associated membrane protein)-GFP (green fluorescent protein) transfection, and HRP uptake were used to quantify various aspects of synaptic vesicle recycling. Our results show that there are very substantial differences in synaptic vesicle recycling at physiological temperature as opposed to the common, lower experimental temperatures. At $37^{\circ} \mathrm{C}$, compared with $23^{\circ} \mathrm{C}$, the speed of both exocytosis and endocytosis was higher. The size of the recycling vesicle pool (in both number of vesicles and spatial extent) was twofold larger at $37^{\circ} \mathrm{C}$. In addition, although repeated $10 \mathrm{~Hz}$ electrical stimulation caused an NMDA receptor-dependent enlargement (averaging 170\%) of the measurable recycling vesicle pool at $23^{\circ} \mathrm{C}$, the same stimulus repetition had no effect at $37^{\circ} \mathrm{C}$. These results show that it is potentially misleading to extend conclusions drawn about vesicle function or presynaptic plasticity at lowered experimental temperature to physiological conditions and that much new experimental work at the higher physiological temperature range will be needed to understand the true parameters of presynaptic functions.
\end{abstract}

Key words: synapse; temperature; exocytosis; endocytosis; recycling vesicle pool; HRP

\section{Introduction}

Recent advances in optical methods have allowed direct measurements of the function and plasticity of individual presynaptic boutons in a variety of species and preparations. The use of fluorescent membrane dyes such as FM 1-43[N-(3-triethylammoniumpropyl)-4(4-(dibutylamino)styryl)pyridinium dibromide] and green fluorescent protein (GFP)-tagged synaptic vesicle proteins have revealed a wealth of information about presynaptic function, such as kinetics of exocytosis and endocytosis of synaptic vesicles, vesicle pool characteristics (for review, see Cochilla et al., 1999; Ryan, 2001), different modes of vesicle recycling (Zenisek et al., 2002; Aravanis et al., 2003; Gandhi and Stevens, 2003), and presynaptic plasticity (Zakharenko et al., 2001, 2002). The development of techniques for photoconversion of FM dyes (Henkel et al., 1996; Harata et al., 2001a) and immunolabeling of GFP (Micheva et al., 2003) have further allowed the extension of such studies to the electron microscope level.

To date, these elegant optical studies have been performed almost exclusively with cell cultures or other explant preparations, and a great majority have been performed at room temper-

Received May 4, 2005; revised June 30, 2005; accepted July 1, 2005.

This work was supported by grants from the National Institutes of Health and by a gift from the Vincent Coates Foundation. We thank Dr. R. W. Tsien for critically reading and discussing this manuscript and Y. Gedde for technical assistance.

Correspondence should be addressed to Kristina D. Micheva, Department of Molecular and Cellular Physiology, Stanford University, 279 Campus Drive, Stanford, CA 94305. E-mail: kmicheva@stanford.edu.

D01:10.1523/JNEUROSCI.1801-05.2005

Copyright $\odot 2005$ Society for Neuroscience $\quad$ 0270-6474/05/257481-08\$15.00/0 ature. Although such conditions are physiological for many lower vertebrate species, these temperatures are at least $10^{\circ} \mathrm{C}$ below physiological for mammalian neurons. It is well known that temperature has large effects on biological phenomena and, in particular, on enzyme kinetics, plasma membrane state, and synaptic transmission (Thompson et al., 1985; Shen and Schwartzkroin, 1988; Volgushev et al., 2000a,b, 2004; Pyott and Rosenmund, 2002). Even synaptic morphology appears to be profoundly affected by temperature changes (Kirov et al., 2004; Roelandse and Matus, 2004). A mammalian synapse cooled down to room temperature may thus function in a very different way than it does at physiological temperature. Therefore, it is particularly important to determine how optical measurements of presynaptic function performed at room temperature relate to a synapse functioning at physiological temperature. Only then can we safely incorporate the new and exciting findings about synaptic function into theories and models of mammalian brain function.

\section{Materials and Methods}

Cell culture and transfection. Primary embryonic hippocampal cultures ("Banker" style) were prepared as described previously (Goslin et al., 1998) and used 7-16 d later. All procedures were approved by the Institutional Animal Care and Use Committee. Neurons were transfected with vesicle-associated membrane protein (VAMP)-GFP (a gift from Dr. R. Scheller, Genentech, South San Francisco, CA) using a modified calcium phosphate transfection protocol (Xia et al., 1996).

Confocal microscopy of live neurons and image analysis. During imaging, neurons were kept at 37 or $23^{\circ} \mathrm{C}$ in Tyrode's solution [containing the 
following (in mM): $\left.119 \mathrm{NaCl}, 2.5 \mathrm{KCl}, 2 \mathrm{CaCl}_{2}, 2 \mathrm{MgCl}_{2}, 25 \mathrm{HEPES}\right], \mathrm{pH}$ 7.4 , with the addition of $30 \mathrm{~mm}$ glucose, $1 \%$ ovalbumin, $10 \mu \mathrm{M}$ 6-cyano7-nitroquinoxaline-2,3-dione (CNQX; Tocris Cookson, Ellisville, MO) and, unless noted otherwise, $50 \mu \mathrm{M} \mathrm{D}(-)$-2-amino-5-phosphonopentanoic acid (D-AP-5; Tocris Cookson). N-(3-triethylammoniumpropyl)-4-(6-(4-(diethylamino)phenyl) hexatrienyl) pyridinium dibromide (FM 4-64) loading was performed by superfusing the dye (5 $\mu \mathrm{M})$ into the imaging chamber and electrically stimulating the neurons by passing current pulses between platinum electrodes placed at opposite ends of the chamber. Temperature control was achieved by circulating air from a precision air heater in a chamber enclosing the microscope stage with the specimen and opening around the objective lens. The air escaping the chamber flows around the objective and controls its temperature as well, thus ensuring constant temperature across the specimen, which is in close thermal contact with the oil-immersion objective.

Imaging was done with a laboratory-designed laser-scanning confocal microscope using a Zeiss (Oberkochen, Germany) $40 \times / 1.3$ numerical aperture Fluar objective. Images were sampled at $0.286 \mu \mathrm{m}$ pixel size and collected every $4.5 \mathrm{~s}$ ( $1 \mathrm{~s}$ in some experiments). Images were analyzed with custom software (N. E. Ziv, Technion, Haifa, Israel). FM 4-64 fluorescence intensities were averaged over $6 \times 6$ pixel squares centered on presynaptic boutons. When analyzing VAMP-GFP-transfected neurons, VAMP-GFP fluorescence intensities were averaged over entire presynaptic boutons and immediately adjacent regions. The area of FM 4-64 fluorescent boutons was obtained using ImageJ. Grayscale images of loaded neurons were converted to black and white images using the Threshold function of the program, and all puncta between 0.14 and 8.00 $\mu \mathrm{m}^{2}$ with a regular rounded shape were analyzed.

HRP uptake and electron microscopy. HRP uptake was achieved by electrically stimulating the neurons $(10 \mathrm{~Hz}$ for $1 \mathrm{~min})$ in the presence of HRP type VI-A (10 mg/ml; Sigma, St. Louis, MO), using the same setup and medium as that for the FM 4-64 loading described above. After the end of stimulation, HRP was left for an additional $1 \mathrm{~min}$ and then washed away. The hippocampal cultures were then fixed in $2.5 \%$ glutaraldehyde in $0.15 \mathrm{M}$ cacodylate buffer for $1 \mathrm{~h}$. HRP was visualized by $25 \mathrm{~min}$ incubation in $3,3^{\prime}$-diaminobenzidine tetrahydrochloride $(0.75 \mathrm{mg} / \mathrm{ml})$ in $0.05 \mathrm{M}$ Tris- $\mathrm{HCl}, \mathrm{pH} 7.6$, containing $0.01 \%$ hydrogen peroxide (Neale et al., 1999). The cultures were then prepared for electron microscopy using rapid microwave irradiation (PELCO 3451 laboratory microwave system; Ted Pella, Redding, CA) and ColdSpot (Ted Pella), as described in detail previously (Micheva et al., 2003). Serial ultrathin sections $(60 \mathrm{~nm})$ were cut with an ultramicrotome (Ultracut E; Leica, Nussloch, Germany), poststained with uranyl acetate and lead citrate, and viewed with a JEM-1230 electron microscope (JEOL, Peabody, MA) at $80 \mathrm{kV}$ accelerated voltage using a Gatan (Pleasanton, CA) 791 CCD camera. ImageJ was used for measurements of synaptic vesicles.

Data analysis. Comparisons between the two experimental temperatures were performed using coverslips from the same neuronal preparation on the same day. Statistical analysis was performed using a two-sided Mann-Whitney test. Data are reported as mean \pm SEM.

\section{Results}

\section{FM 4-64 loading detects a larger recycling vesicle pool at physiological temperature}

The FM fluorescent membrane dyes are routinely used for the determination of synaptic vesicle pool sizes. When applied extracellularly during periods of synaptic activity, FM dyes become trapped within recycled synaptic vesicles (FM loading) and can be visualized by fluorescence microscopy after washing off extracellular dye. The intensity of the trapped dye is proportional to the number of recycled vesicles. Subsequent exocytosis causes release of the dye (FM unloading) (Betz et al., 1992).

We loaded hippocampal synapses with FM 4-64 using electrical stimulation at $10 \mathrm{~Hz}$ for $1 \mathrm{~min}$, and the dye was left for an additional $1 \mathrm{~min}$ before washing out. This protocol labels $\sim 90 \%$ of the total recycling vesicle pool at room temperature (Ryan and Smith, 1995). Subsequently, the synapses were unloaded by elec- trical stimulation at $10 \mathrm{~Hz}$ for $2 \mathrm{~min}$, and the FM 4-64 labeling of synapses was determined as the difference in intensity of the synapses in their loaded and unloaded state. Under these conditions, the fluorescence intensity of the FM 4-64 labeling was significantly higher at $37^{\circ} \mathrm{C}[660 \pm 16$ arbitrary units (a.u.); $n=941$ boutons from nine coverslips] compared with $23^{\circ} \mathrm{C}$ ( $412 \pm 9$ a.u.; $n=1281$ boutons from 10 coverslips; $p<0.0001$; Mann-Whitney test) (Fig. $1 A, B$ ). In addition, the area of the FM 4-64 puncta was significantly larger at $37^{\circ} \mathrm{C}$ (Fig. $1 C$ ). Thus, at $37^{\circ} \mathrm{C}$, the apparent spatial extent of individual FM 4-64-labeled vesicle pools (measured as punctum area) averaged $1.61 \pm 0.06 \mu \mathrm{m}^{2}(n=634$ boutons from two coverslips) compared with $1.10 \pm 0.04 \mu \mathrm{m}^{2}$ at $23^{\circ} \mathrm{C}$ ( $n=673$ boutons from two coverslips). Longer loading protocols (e.g., $2 \mathrm{~min}$ at $10 \mathrm{~Hz}$ in the presence of the dye and 1 min waiting period before washing off the dye) resulted in similar differences between the two temperatures but with an increased background (results not shown).

Fluorescence emission characteristics of many dyes are known to depend on temperature, and, as the temperature is increased, the fluorescence decreases, a phenomenon known as temperature quenching (Guibault, 1990). The change in fluorescence is normally $1 \%$ per $1^{\circ} \mathrm{C}$; however, for some compounds, it can be as high as $5 \%$ per $1^{\circ} \mathrm{C}$. We found that the fluorescence of FM 4-64 molecules in membranes decreases fairly substantially with increasing temperature. To quantify this relationship, dye-loaded presynaptic boutons were imaged while alternating the temperature of the medium between 23 and $37^{\circ} \mathrm{C}$. The FM 4-64 signal at $37^{\circ} \mathrm{C}$ was, on average, $70 \pm 2 \%$ of the signal at $23^{\circ} \mathrm{C}$. Similar results were obtained by imaging larger areas of cell bodies or dendrites, the plasma membrane of which is also weakly stained after exposure to FM 4-64 (67 $\pm 4 \%$ fluorescent signal at $37^{\circ} \mathrm{C}$ ). Paraformaldehyde fixation of the cultures immediately after dye loading did not affect the temperature dependence of FM 4-64 $\left(71 \pm 2 \%\right.$ fluorescent signal at $\left.37^{\circ} \mathrm{C}\right)$, suggesting that these temperature effects depend on intrinsic properties of the dye in the membrane environment. Therefore, the actual difference in the total recycling vesicle pool at the two temperatures is expected to be substantially larger than the 1.6 times difference apparent from uncorrected FM 4-64 measurements. Thus, our data suggest that the recycling vesicle membrane pool may be approximately two times larger at $37^{\circ} \mathrm{C}$ compared with $23^{\circ} \mathrm{C}$.

\section{Electron microscopic characterization of the recycling vesicle pools: morphology}

To directly confirm the above conclusions about temperaturerelated changes in the total vesicle recycling pool, we loaded presynaptic boutons with HRP using the same stimulation protocol as that for FM 4-64 and then processed the neurons for electron microscopy. HRP, detected by the dark appearance of its DAB reaction product, was found predominantly in synaptic vesicles, clathrin-coated vesicles, and other membranous structures in presynaptic terminals (Figs. 2, 3). HRP was also observed occasionally in larger vesicular structures (both uncoated and clathrin coated) in dendrites and cell bodies. Meanwhile, in control experiments, when neurons were incubated in HRP without electrical stimulation, only an occasional presynaptic vesicle was labeled at both temperatures $\left(1.9 \pm 0.6 \%\right.$ at $23^{\circ} \mathrm{C}$ and $2.2 \pm 0.8 \%$ at $37^{\circ} \mathrm{C}$, as counted on single-section presynaptic bouton profiles; $n=20$ for $23^{\circ} \mathrm{C} ; n=13$ for $37^{\circ} \mathrm{C}$.).

To quantify the differences in synaptic pools at the two temperatures, we compared the size distribution of HRP-positive structures by measuring their surface area on single sections (Fig. 2 ). As seen from the frequency distribution histogram of the data 

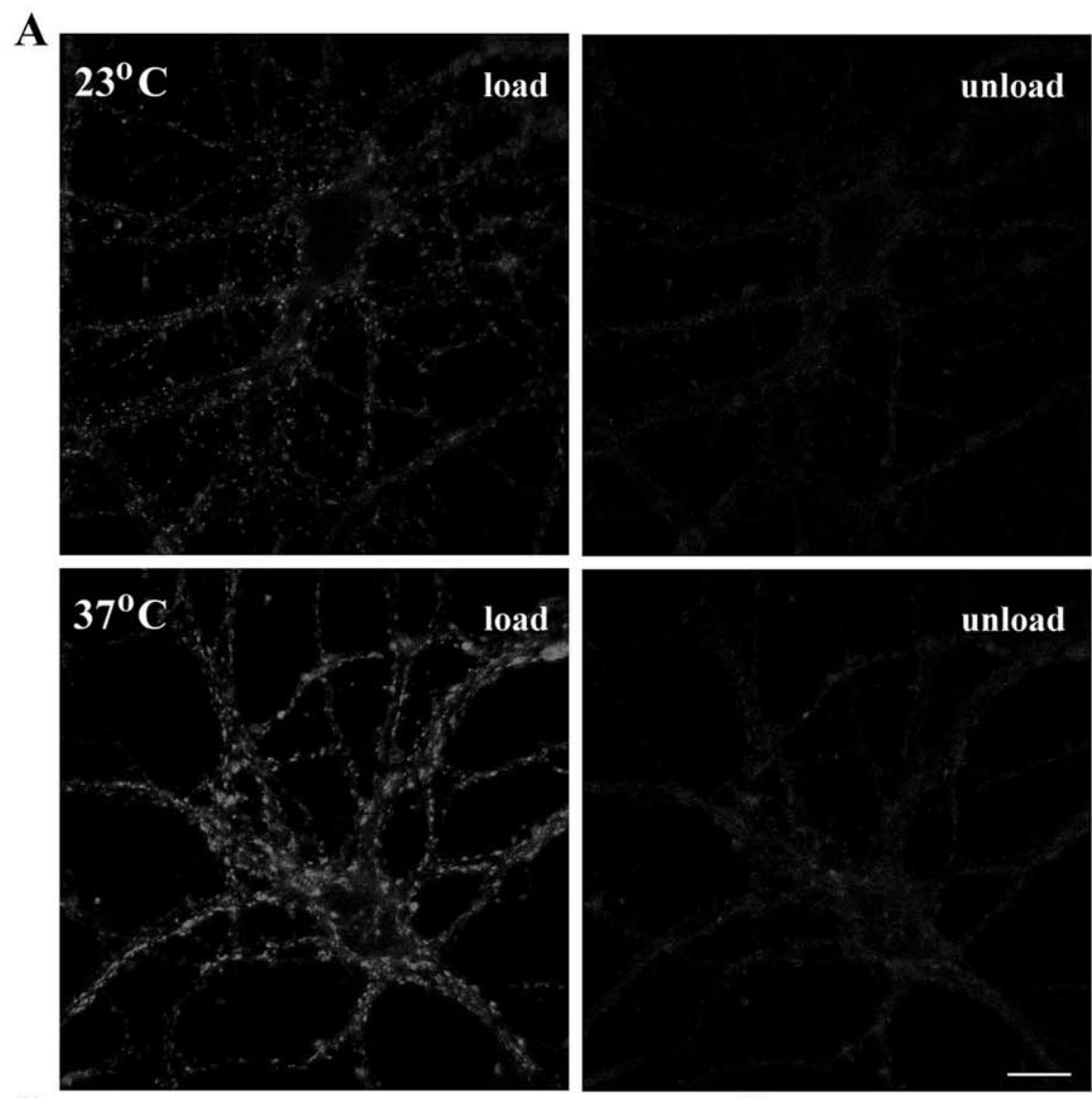

B

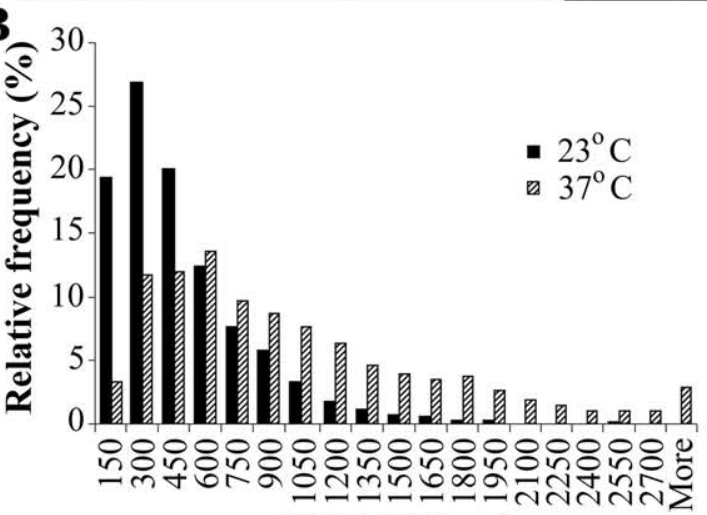

FM 4-64 (a.u.)

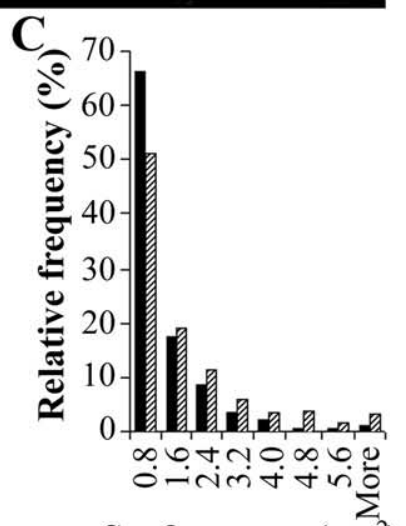

Surface area $\left(\mu \mathrm{m}^{2}\right)$

Figure 1. FM 4-64 loading of hippocampal neurons in culture. $\boldsymbol{A}$, Confocal images of hippocampal neurons in primary culture (12 d in vitro) loaded with FM 4-64 (left) and subsequently unloaded (right). The neurons were kept at either room temperature (top) or physiological temperature (bottom). Loading was achieved using electrical stimulation at $10 \mathrm{~Hz}$ for $1 \mathrm{~min}$ and unloading at $10 \mathrm{~Hz}$ for $2 \mathrm{~min}$. Scale bar, $10 \mu \mathrm{m}$. B. Histogram presenting the distribution of the fluorescence intensity of the FM 4-64 labeling at individual synapses at both temperatures; fluorescence values are corrected for temperature quenching $(n=1281$ boutons from 10 coverslips at $23^{\circ} \mathrm{C}$, and $n=941$ boutons from 9 coverslips at $37^{\circ} \mathrm{C}$ ). C, Distribution of the surface area of individual FM 4-64 puncta at both temperatures ( $n=673$ boutons from 2 coverslips at $23^{\circ} \mathrm{C}$, and $n=634$ boutons from 2 coverslips at $37^{\circ} \mathrm{C}$ ).

(Fig. $2 B$ ), the great majority of HRP vesicles had an area falling within the range of the area of clear synaptic vesicles $(35-50 \mathrm{~nm}$ in diameter corresponding to $\sim 1000-2000 \mathrm{~nm}^{2}$ ). The population of larger HRP vesicles consisted of a variety of endocytic vesiculotubular structures, and they were more frequently observed at $37^{\circ} \mathrm{C}$. Thus, $11 \%$ of the HRP vesicles had an area $>2000 \mathrm{~nm}^{2}$ at $23^{\circ} \mathrm{C}$ compared with $16 \%$ at $37^{\circ} \mathrm{C}$. Overall, the average area of $\mathrm{HRP}$ vesicles was significantly increased at $37^{\circ} \mathrm{C}\left(1517 \pm 41 \mathrm{~nm}^{2}\right.$ at $37^{\circ} \mathrm{C}$ compared with $1318 \pm 32 \mathrm{~nm}^{2}$ at $23^{\circ} \mathrm{C} ; p<0.01$; Mann-Whitney).
Electron microscopy confirms the twofold increase in total recycling vesicle pool at

\section{physiological temperature}

Additional analysis was performed on serially sectioned synaptic boutons, where the following data were obtained: number of HRP synaptic vesicles (i.e., area $<2000$ $\mathrm{nm}^{2}$ ), number of larger HRP structures, number of non-HRP synaptic vesicles, and total number of synaptic vesicles per bouton (Fig. 3). A total of 82 boutons from two coverslips were analyzed at $23^{\circ} \mathrm{C}$, and 89 boutons from two coverslips were analyzed at $37^{\circ} \mathrm{C}$. There were twice as many $\mathrm{HRP}$ synaptic vesicles per bouton at $37^{\circ} \mathrm{C}$ (89 \pm 7 HRP synaptic vesicles) compared with $23^{\circ} \mathrm{C}(43 \pm 4 \mathrm{HRP}$ synaptic vesicles; significantly different at $p<0.0001$; Mann-Whitney test). In addition, more larger endocytic structures labeled with HRP were observed at $37^{\circ} \mathrm{C}(19 \pm 2$ per bouton at $37^{\circ} \mathrm{C}$ compared with $6 \pm 1$ at $23^{\circ} \mathrm{C}$; $p<0.0001$; Mann-Whitney test). At both temperatures, the number of larger HRP structures was positively correlated with the number of HRP synaptic vesicles (for $23^{\circ} \mathrm{C}$, Spearman's rank correlation coefficient, $r_{\mathrm{s}}=0.59, p<0.000001$; for $37^{\circ} \mathrm{C}$, $\left.r_{\mathrm{s}}=0.65, p<0.000001\right)$. The larger HRP structures are probably either endosomes labeled by HRP from one or more endocytic vesicles fusing with it or may represent portions of the plasma membrane internalized by bulk endocytosis (De Camilli et al., 2001). In either case, they would correspond to at least one exocytosed synaptic vesicle, and thus, the sum of the small HRP-containing synaptic vesicles and larger HRP structures will represent the lower limit of the total number of recycling vesicles. Calculated in this manner, the total number of recycling vesicles is at least 108 at physiological temperature and 49 at room temperature.

The number of non-HRP clear synaptic vesicles per bouton was larger at $23^{\circ} \mathrm{C}(175$ \pm 28 compared with $113 \pm 7$ at $37^{\circ} \mathrm{C} ; p=$ 0.051; Mann-Whitney test). The total number of synaptic vesicles was similar at both temperatures $\left(219 \pm 29\right.$ at $23^{\circ} \mathrm{C}$ and $202 \pm 12$ at $\left.37^{\circ} \mathrm{C}\right)$. These results from the EM analysis are in agreement with our observations using FM dyes and directly confirm that the same
stimulus can recruit twice the number of synaptic vesicles at physiological temperature compared with room temperature.

In addition, our EM results underscore other important temperature-dependent differences of synaptic vesicle recycling that cannot be easily detected using confocal imaging of FM dyes. Thus, $\sim 5 \%$ of synapses ( 4 of 82 ) have less than or equal to five HRP vesicles at $23^{\circ} \mathrm{C}$, but none of the 89 synapses observed at $37^{\circ} \mathrm{C}$ was with such a small number of recycled vesicles. At $37^{\circ} \mathrm{C}$, the great majority of synapses $(96 \% ; 85$ of 89$)$ use $>25$ vesicles 
when stimulated at $10 \mathrm{~Hz}$ for $1 \mathrm{~min}$, but this percentage drops to $66 \%$ (54 of 82 ) at $23^{\circ} \mathrm{C}$.

\section{Docked vesicles}

The number of docked vesicles, defined as synaptic vesicles in direct contact with the presynaptic plasma membrane at the active zone, was counted in a subset of boutons in which the plane of section was approximately perpendicular to the active zone. The number of docked HRP vesicles per active zone was not different at the two temperatures $\left(2.3 \pm 0.3\right.$ at $23^{\circ} \mathrm{C}, n=46$; $2.6 \pm 0.3$ at $\left.37^{\circ} \mathrm{C}, n=53\right)$. However, the number of docked non-HRP vesicles was almost twice as high at $23^{\circ} \mathrm{C}(6.8 \pm 1.0$ vs $3.8 \pm 0.4$ at $37^{\circ} \mathrm{C} ; p=0.0375$; MannWhitney test) The total number of all docked vesicles tended to be higher at $23^{\circ} \mathrm{C}$ with $9.0 \pm 1.3$ docked vesicles per active zone compared with $6.5 \pm 0.5$ vesicles at $37^{\circ} \mathrm{C}$, but this trend did not reach statistical significance ( $p=0.27$; Mann-Whitney test).

\section{Synaptic vesicle exocytosis is faster and less variable at physiological temperature}

The fluorescent membrane dye FM 4-64 was also used to compare exocytosis kinetics at 37 and $23^{\circ} \mathrm{C}$. Hippocampal neurons were loaded by electrical stimulation at 10 $\mathrm{Hz}$ for $30 \mathrm{~s}$, and, after an additional $30 \mathrm{~s}$, the dye was washed away. FM unloading was achieved by electrical stimulation at 10 $\mathrm{Hz}$ for 2 min. Normalized FM unloading curves (Fig. 4) show that, at room temperature, exocytosis proceeds more slowly and is much more variable compared with physiological temperature. The actual difference is even bigger, because the recycling vesicle pool is more than two times larger at $37^{\circ} \mathrm{C}$, as shown above. Using the normalized FM fluorescence data (Fig. 4C, the second time point after the beginning of stimulation) and the EM data about the number of recycling synaptic vesicles, we can calculate the absolute speed of exocytosis, assuming that there is no vesicle reuse in the initial period of stimulation. Thus, during the first $9 \mathrm{~s}$ of electrical stimulation, an average presynaptic bouton releases 49 synaptic vesicles at $37^{\circ} \mathrm{C}$ (i.e., $45 \%$ of 110 vesicles), compared with 19 vesicles at $23^{\circ} \mathrm{C}$ (i.e., $38 \%$ of 50 vesicles). This amounts to a difference of 2.6 times in the absolute speed of exocytosis at the two temperatures.

\section{Synaptic vesicle endocytosis is faster at physiological temperature}

Synaptic vesicle endocytosis was quantified by transfecting the neurons with a cDNA construct encoding VAMP2/synaptobrevin fused to GFP. The fusion protein is oriented such that GFP is

C
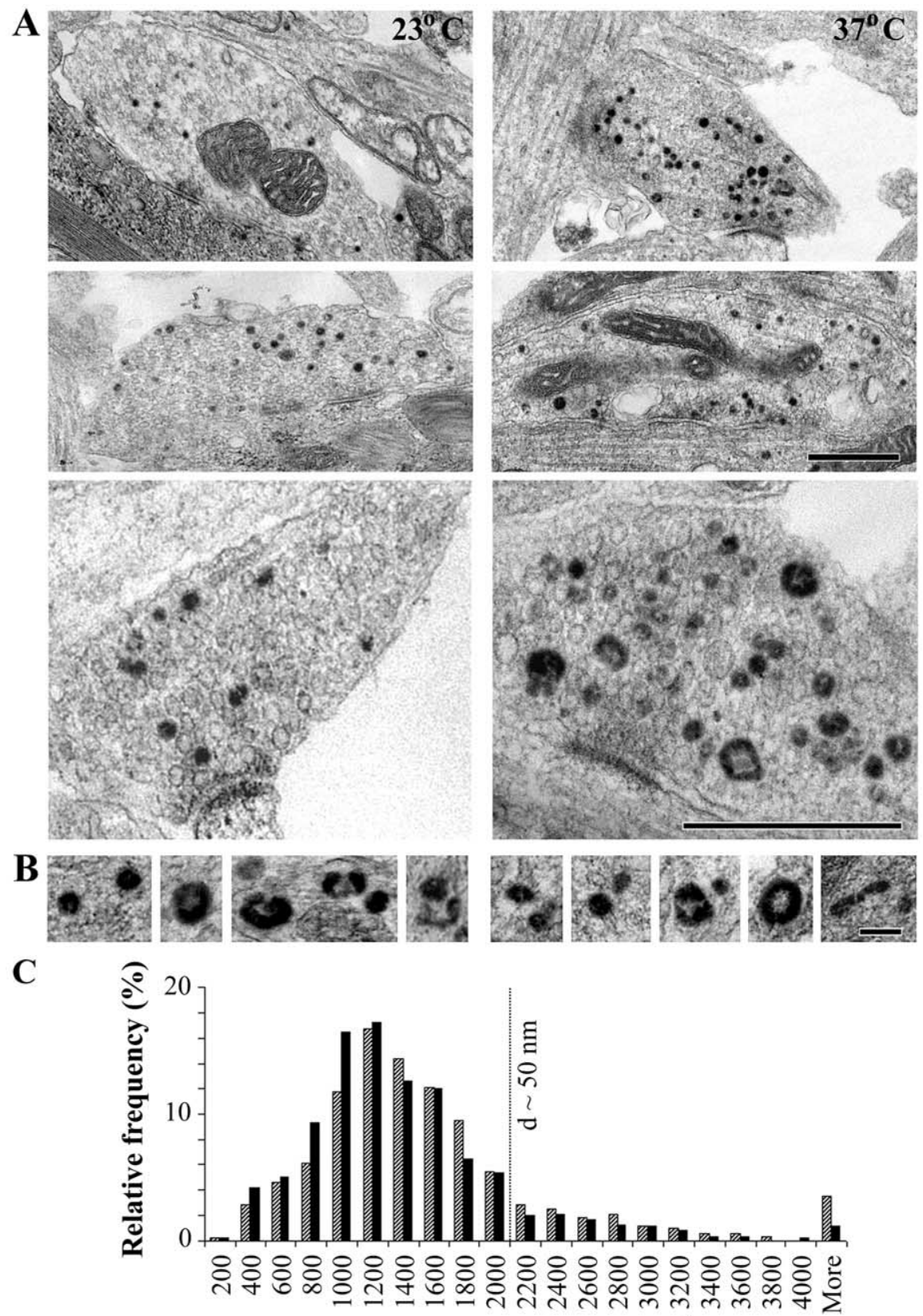

\section{Area of HRP vesicles $\left(\mathrm{nm}^{2}\right)$}

Figure 2. Morphology of HRP-labeled structures after electrical stimulation. $A$, Electron micrographs of presynaptic boutons formed by hippocampal neuronal cultures ( $15 \mathrm{~d}$ in vitro) electrically stimulated in the presence of HRP at $23^{\circ} \mathrm{C}$ and $37^{\circ} \mathrm{C}$. HRP uptake is identified by the dark DAB reaction product. Scale bar, $0.5 \mu \mathrm{m}$. $\boldsymbol{B}$, Examples of HRP-labeled structures at the two temperatures. Scale bar, $100 \mathrm{~nm}$. $C$, Distribution of the area of HRP vesicles (in square nanometers) as measured on single sections ( $n=603$ vesicles at $23^{\circ} \mathrm{C}$, and $n=610$ vesicles at $37^{\circ} \mathrm{C}$ ). d, Diameter.

within the acidic lumen of synaptic vesicles. Because of its $\mathrm{pH}$ sensitivity, GFP fluorescence increases after exocytosis when VAMP-GFP becomes exposed to the neutral extracellular $\mathrm{pH}$. Subsequent endocytosis of synaptic vesicles is reported quantitatively by a decrease in GFP fluorescence, caused by internalization of VAMP-GFP within endocytic vesicles and their rapid acidification (Li and Murthy, 2001). In contrast to FM 4-64, VAMP-GFP fluorescence and its dependence on $\mathrm{pH}$ did not change with temperature, as confirmed with a series of control experiments. Transfected hippocampal neurons were fixed and permeabilized, and VAMP-GFP fluorescence was measured at 


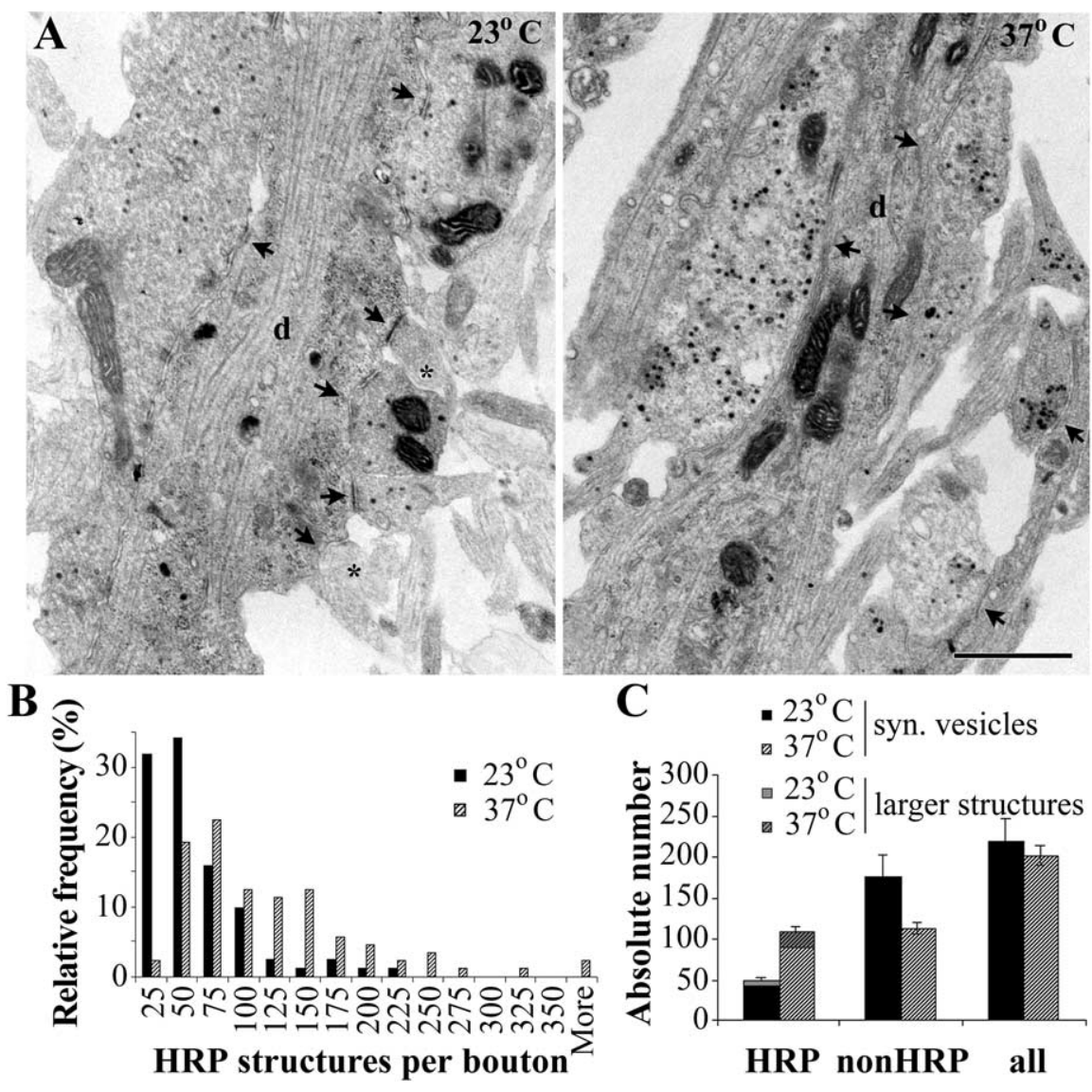

Figure 3. Recycling vesicle pool in hippocampal neurons identified by $H R P$ uptake. $A, A t 23^{\circ} \mathrm{C}$, compared with $37^{\circ} \mathrm{C}$, a smaller number of HRP vesicles is observed, and some presynaptic profiles are even entirely devoid of HRP vesicles (asterisk). The arrows indicate presynaptic boutons. d, Dendrite. Scale bar, $0.5 \mu \mathrm{m}$. $\boldsymbol{B}$, Distribution of the number of HRP structures per presynaptic bouton, as determined from serial sections. $C$, Absolute numbers of HRP, non-HRP, and all vesicles per presynaptic bouton (mean $\pm \mathrm{SE} ; n=82$ boutons from 2 coverslips at $23^{\circ} \mathrm{C}$, and $n=89$ boutons from 2 coverslips at $37^{\circ} \mathrm{C}$ ). syn., Synaptic. Error bars represent SEM.

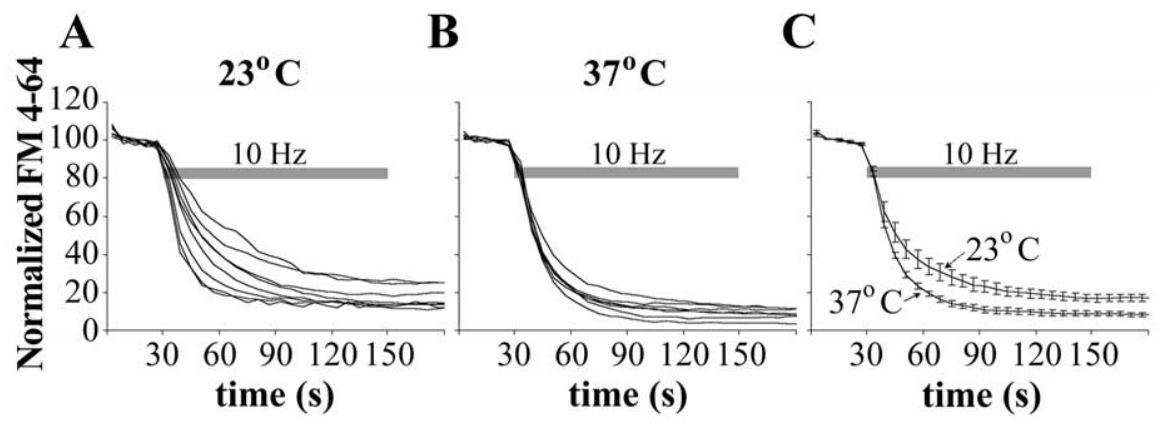

Figure 4. Speed of exocytosis as measured with FM 4-64. $\boldsymbol{A}, \boldsymbol{B}$, Unloading curves from experiments performed at $23^{\circ} \mathrm{C}(\boldsymbol{A} ; n=$ 8 coverslips with at least 100 boutons each) and $37^{\circ} \mathrm{C}(\boldsymbol{B} ; n=7$ coverslips with at least 100 boutons each). FM 4-64 fluorescence intensities are normalized to the initial resting state of each presynaptic bouton and averaged for each experiment. SEs are not presented in $\boldsymbol{A}$ and $\boldsymbol{B}$, because they were on the order of $1 \%$. C, Comparison of the mean unloading curves from the experiments in $\boldsymbol{A}$ and $\boldsymbol{B}$ with SEs.

varying temperatures and $\mathrm{pH}$. VAMP-GFP fluorescence did not change from 37 to $23^{\circ} \mathrm{C}$, and the ratio between the VAMP-GFP fluorescence at $\mathrm{pH} 7.4$ and $\mathrm{pH} 5.6$ (corresponding to the $\mathrm{pH}$ of the synaptic vesicle lumen) remained stable (2.3 ratio).

For the quantification of synaptic vesicle endocytosis, hippocampal neurons were transfected with VAMP-GFP and loaded with FM 4-64 by electrical stimulation for $30 \mathrm{~s}$ at $10 \mathrm{~Hz}$ to help identify active synapses. Electrical stimulation of the neurons $(2 \mathrm{~min}$ at $10 \mathrm{~Hz}$ ) evoked synaptic vesicle exocytosis, which resulted in an increase in presynaptic VAMP-GFP fluorescence. Fluorescence gradually returned to baseline after the end of stimulation, presumably because of endocytosis and reacidification of vesicles. The time courses of VAMP-GFP intensity changes in presynaptic boutons were compared at the two temperatures (Fig. 5). Electrical stimulation resulted in a bigger increase in VAMP-GFP fluorescence at $37^{\circ} \mathrm{C}$, consistent with the larger recycling vesicle pool described above. The difference in the peak VAMP-GFP fluorescence at the two temperatures, however, was not as big as the previously measured difference in the recycling vesicle pool, because the VAMPGFP fluorescence detects the balance of exocytosis and endocytosis, and endocytosis proceeds faster at physiological temperature, as described below. After the end of electrical stimulation, VAMP-GFP fluorescence returned to baseline faster at $37^{\circ} \mathrm{C}$ (time constant of $107 \pm 7 \mathrm{~s}$ ) compared with $23^{\circ} \mathrm{C}$ (time constant of $153 \pm 10 \mathrm{~s}$; $p<0.001$; Mann-Whitney test). Bearing in mind that more than twice the number of synaptic vesicles have to be recycled at $37^{\circ} \mathrm{C}$, this result points to a significant, approximately threefold increase in the absolute speed of endocytosis after electrical stimulation at physiological temperature.

\section{A form of NMDA-dependent presynaptic potentiation can only be detected at $23^{\circ} \mathrm{C}$}

We observed from previous experiments at $37^{\circ} \mathrm{C}$ (Micheva et al., 2003) that, when synaptic boutons are tested twice serially with the same FM 4-64 protocol (30 s at 10 $\mathrm{Hz}$ load; $2 \mathrm{~min}$ at $10 \mathrm{~Hz}$ unload), they take up equal amounts of the dye, even when the second loading session closely follows the first $(<2 \mathrm{~min})$. To our surprise, when the same experiment was repeated at $23^{\circ} \mathrm{C}$, synaptic boutons loaded significantly more dye the second time (Fig. 6A). Thus, although the ratio of the second-to-first load was $1.03 \pm 0.04$ at $37^{\circ} \mathrm{C}$, it reached $1.69 \pm 0.17$ at $23^{\circ} \mathrm{C}(p<0.001$; MannWhitney test). The extent of this potentiation was quite variable, exceeding on a few occasions a ratio of 3.00. These experiments were done in the presence of CNQX (AMPA/kainate antagonist) but without D-AP-5 (NMDA antagonist). The addition of D-AP-5 abolished the presynaptic potentiation at $23^{\circ} \mathrm{C}$, without having an effect on the loading at $37^{\circ} \mathrm{C}$ (Fig. $6 B-D$ ). Therefore, although at $23^{\circ} \mathrm{C}$, a $10 \mathrm{~Hz}$ stimulus can induce a pronounced NMDA-dependent presynaptic potentiation, this phenomenon completely disappears at physiological temperature.

These results can be interpreted that either the NMDA- 


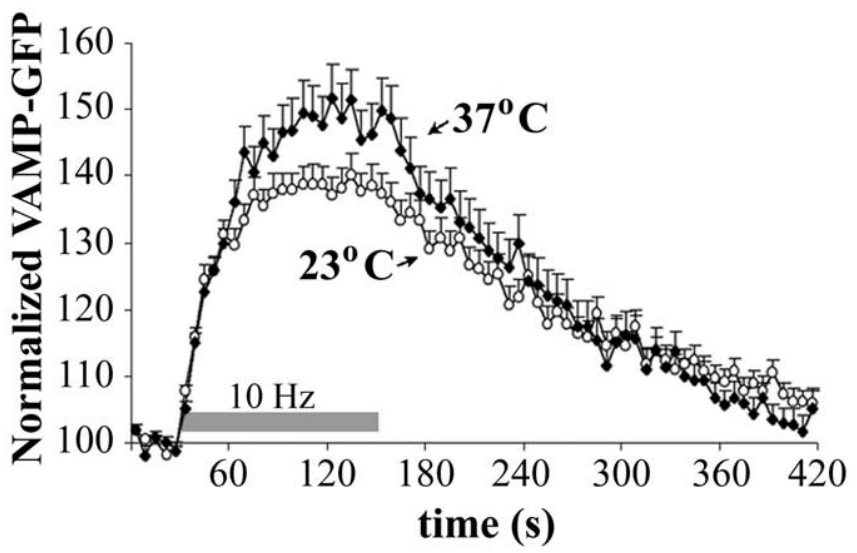

Figure 5. Speed of endocytosis determined with VAMP-GFP. VAMP-GFP fluorescence changes in presynaptic boutons during electrical stimulation at 23 and $37^{\circ} \mathrm{C}$ are shown. Mean values of the normalized VAMP-GFP fluorescence intensities and SEs are shown (for $23^{\circ} \mathrm{C} n=$ 222 boutons from 7 coverslips and $37^{\circ} \mathrm{C}, n=157$ boutons from 7 coverslips).

dependent presynaptic potentiation, which was described previously in neuronal cultures (Ryan et al., 1996), is an artifact of the lowered experimental temperature, or the threshold for inducing presynaptic plasticity is temperature dependent. To test these possibilities, we repeated the above experiments at $37^{\circ} \mathrm{C}$ in the absence of D-AP-5 using a stronger $50 \mathrm{~Hz}$ unloading stimulus instead of $10 \mathrm{~Hz}$. Under these conditions, presynaptic potentiation was induced in 5 of 11 coverslips (ratio of second-to-first load, $1.37 \pm 0.05$ ). This potentiation, however, was not as strong as the one induced at $23^{\circ} \mathrm{C}$ with a $10 \mathrm{~Hz}$ stimulus, as described above, where all of the 16 coverslips tested displayed some degree of potentiation. Thus, lowering the experimental temperature appears to decrease the threshold for inducing presynaptic plasticity.

\section{Discussion}

Our results show that temperature is a crucial experimental factor in studies of mammalian synapses, because it substantially influences the speed, magnitude, and even the nature of presynaptic events. At physiological temperature compared with room temperature, the size of the recycling vesicle pool is two times larger, and the speed of both exocytosis and endocytosis is faster. In addition, a form of NMDA-dependent presynaptic plasticity that is prominent at room temperature cannot be observed at physiological temperature. Here, it should be noted that our methods are not designed to detect rapid exocytic/endocytic events, such as "kiss-and-run," in which vesicles cannot be stained by FM dyes or HRP and thus we do not provide information on vesicles that may be possibly involved in such pathways.

A recent study of hippocampal neurons in culture (Fernandez-Alfonso and Ryan, 2004) also highlighted the temperature dependence of presynaptic function and conclusively showed that, at physiological temperature, a faster endocytosis is sufficient to balance exocytosis and to avoid vesicle pool depletion. There are, however, some differences with our results, such as the surprisingly small increase $(\sim 10 \%)$ in the recycling vesicle pool, as well as a decrease in the speed of exocytosis at physiological temperature. Apart from the different methods used in both studies, the fluorescence data were also analyzed differently. Fernandez-Alfonso and Ryan (2004) measured the fluorescence of a small central region of individual boutons $\left(0.4 \times 0.4 \mu \mathrm{m}^{2}\right)$, whereas we used larger regions $\left(1.7 \times 1.7 \mu \mathrm{m}^{2}\right)$ corresponding to the average size of FM 4-64 puncta or, for the VAMP-GFP mea-
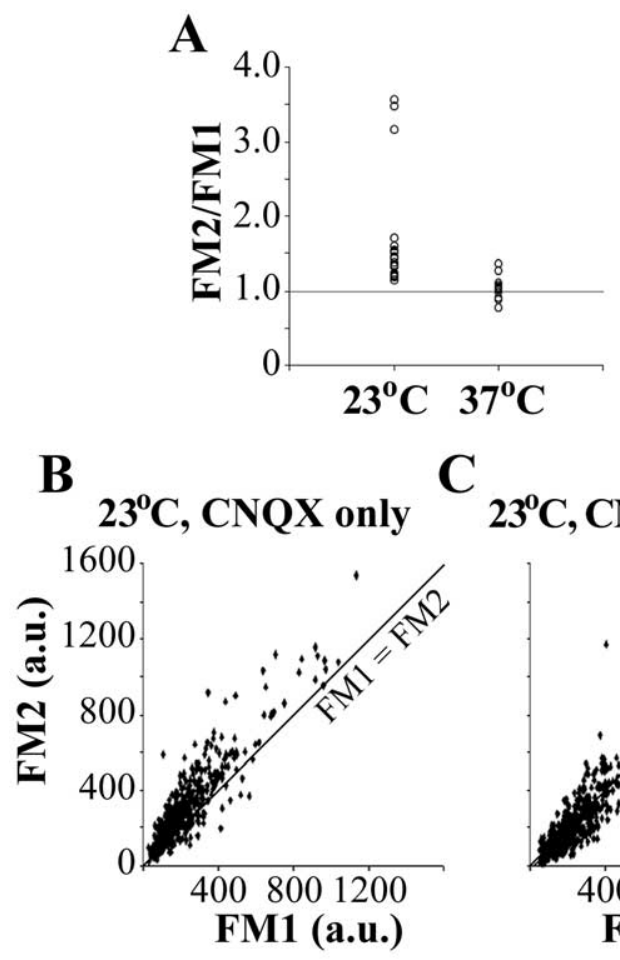

$23^{0} \mathrm{C}, \mathrm{CNQX}+\mathrm{D}-\mathrm{AP5}$
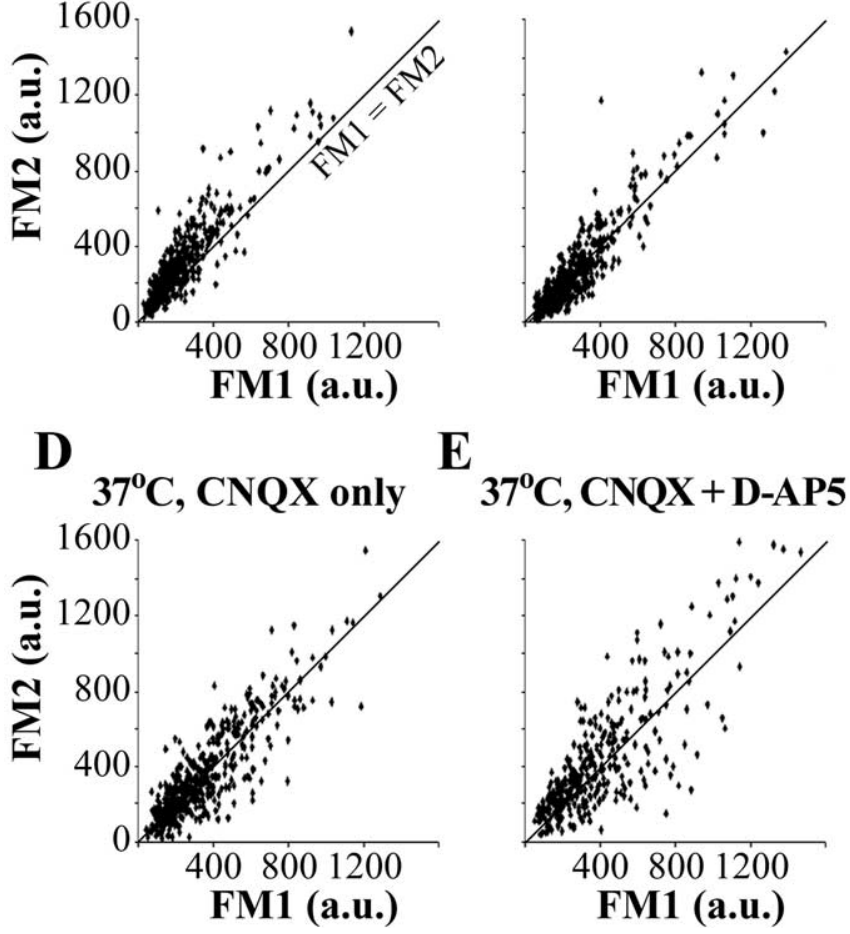

E

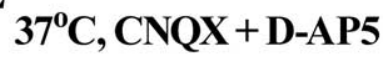

Figure 6. Presynaptic potentiation of hippocampal neurons. A, Synaptic boutons were loaded twice serially with the same stimulation protocol. Mean values from the second FM load normalized to the first load are presented ( $n=16$ coverslips at $23^{\circ} \mathrm{C}$ and $n=13$ coverslips at $\left.37^{\circ} \mathrm{C}\right) . \boldsymbol{B}-\boldsymbol{E}$, Temperature and NMDA receptor dependence of the presynaptic potentiation. Second FM load versus first FM load for individual presynaptic boutons stimulated at 23 or $37^{\circ} \mathrm{C}$, in the presence or absence of an NMDA blocker (D-AP-5; $50 \mu \mathrm{m}$ ), is shown. For each plot, $n \geq$ 150 boutons from two coverslips. The line represents FM1 = FM2. Potentiation is evident only at $23^{\circ} \mathrm{C}$ in the absence of $\mathrm{D}-\mathrm{AP}-5$, where most of the data points are above the FM1 $=F M 2$ line.

surements, also encompassing the immediately adjacent to the bouton areas. Because the size of FM 4-64 puncta (i.e., the area occupied by recycling vesicles) changes with temperature, these two different ways of analysis may yield different results. It is also important to keep in mind that the effect of temperature on synaptic function is the result of the temperature dependence of a great number of factors that could affect synaptic transmission in either direction, and thus slight differences in the experimental conditions may lead to opposite effects (Volgushev et al., 2000a,b). Regarding the speed of exocytosis, a recent electrophysiological study using autaptic cultures of hippocampal neurons appears to support our findings. Thus, improved release capacity was observed at higher temperature, caused by an increased refilling rate of the readily releasable pool and decreased release probability (Pyott and Rosenmund, 2002). 


\section{The size of the recycling vesicle pool}

Mammalian CNS presynaptic boutons widely differ in their size and synaptic vesicle content. In the hippocampus, there are, on average, $\sim 200$ synaptic vesicles per bouton (Schikorski and Stevens, 1997 and present study). Extensive stimulation mobilizes only part of these vesicles, known as the recycling vesicle pool, whereas the remaining vesicles have been termed the "resting pool” (Südhof, 2004). Most previous studies have been conducted at room temperature and have reported surprisingly small recycling vesicle pool sizes: 25 vesicles (Murthy and Stevens, 1999) and 30 - 45 vesicles (Harata et al., 2001b), and this idea now appears to be generally accepted, although substantially larger pools (average, 127 vesicles) (Ryan et al., 1997) have been described at similar low temperatures. The assumed small size of the recycling vesicle pool, together with the relatively slow speed of clathrin-mediated endocytosis as identified at room temperature, appear difficult to reconcile with the sustainability of neurotransmitter release, and this contradiction has been a major motivator in searching for faster modes of synaptic vesicle recycling (Harata et al., 2001b; Aravanis et al., 2003; Gandhi and Stevens, 2003).

Our present results from the FM 4-64 loading experiments and the EM analysis of HRP uptake indicate that the recycling vesicle pool is approximately two times larger at physiological temperature, with at least 110 recycling vesicles per presynaptic bouton, compared with 50 at $23^{\circ} \mathrm{C}$. Also, we observed that the speed of endocytosis is significantly faster at physiological temperature, as described previously in a variety of mammalian secretory cells, including neurons (Mamdouh et al., 1996; Dinkelacker et al., 2000; Pyott and Rosenmund, 2002; FernandezAlfonso and Ryan, 2004). The larger size of the recycling vesicle pool and the higher speed at which it can be reused via the "classical" clathrin-mediated mode of endocytosis considerably increase the theoretical upper limit for maximum sustained release rate at a mammalian CNS synapse at physiological temperature. And although these arguments do not bear directly on the possible existence of additional modes of synaptic vesicle recycling, such as the kiss-and-run mode, they do suggest that alternative accelerated modes may not be necessary to account for physiologically observed release rates. Moreover, the substantial temperature-related differences in synaptic function reported here imply that meaningful future tests for alternative modes of vesicle recycling must be performed at physiological temperature. It may be of interest that recent studies of vesicle recycling in lower vertebrates (performed at their lower physiological temperatures) have yielded results that do not favor a kiss-and-run mechanism (Zenisek et al., 2002; Rizzoli and Betz, 2004).

\section{The size of the readily releasable pool}

The readily releasable pool of synaptic vesicles (i.e., the vesicles that are immediately available for release) is usually equated to the number of docked vesicles (for review, see Regehr and Stevens, 2001) [but see Rizzoli and Betz (2004) for the frog neuromuscular junction]. Our estimate of the total number of docked vesicles is within the previously reported range (Harris and Sultan, 1995; Schikorski and Stevens, 1997). No statistically significant temperature-dependent changes in the number of docked vesicles were observed. This is consistent with electrophysiological measurements of the readily releasable pool at physiological and room temperatures (Pyott and Rosenmund, 2002). It would thus appear that a major variable susceptible to temperature changes is the refill rate but not the size of the readily releasable pool. At both temperatures, the proportion of HRP vesicles in the docked pool $\left(28 \%\right.$ at $23^{\circ} \mathrm{C}$ and $43 \%$ at $\left.37^{\circ} \mathrm{C}\right)$ was very similar to the proportion of HRP vesicles in the total pool $\left(24 \%\right.$ at $23^{\circ} \mathrm{C}$ and $43 \%$ at $\left.37^{\circ} \mathrm{C}\right)$, suggesting that newly recycled vesicles are just as likely to be released during subsequent exocytosis events as the other synaptic vesicles. This ultrastructural observation is consistent with the previously reported intermixing of vesicles that occurs with intense stimulation (Ryan and Smith, 1995; Murthy and Stevens, 1999; Pyle et al., 2000). However, we cannot exclude the possibility that the non-HRP vesicles in the docked pool could be the morphological correlate of rapidly recycling vesicles (Pyle et al., 2000; Aravanis et al., 2003; Ghandhi and Stevens, 2003), which are open to the extracellular environment for a very brief time $(\leq 1 \mathrm{~s})$ insufficient to allow HRP labeling. If this were the case, such rapidly recycling vesicles would be almost twice as prevalent at room temperature (seven per active zone) compared with physiological temperature (four per active zone).

\section{Temperature-related changes in plasticity}

Synaptic plasticity is a fascinating topic full of unresolved controversies (Malenka and Siegelbaum, 2001; Lisman, 2003; Silva, 2003; Lynch, 2004). An important factor contributing to these controversies may be the experimental temperature. Thus, our present results show that a stimulus that has no effect on synaptic function at physiological temperature can cause profound NMDA-dependent presynaptic changes at room temperature. Consistent with our results, a weak tetanus led to a much stronger NMDA-dependent potentiation of Schaffer collaterals in hippocampal slices when applied at $23^{\circ} \mathrm{C}$ compared with $32^{\circ} \mathrm{C}(\mathrm{Mc}-$ Naughton et al., 1994). This temperature-dependent variability in synaptic plasticity may be attributable to a number of concurrent changes occurring at room temperature, including broadening of action potentials (Thompson et al., 1985; Shen and Schwartzkroin, 1988; Volgushev et al., 2000a,b), larger $\mathrm{Ca}^{2+}$ influx produced by an action potential (Borst and Sakmann, 1998; David and Barrett, 2000; Dinkelacker et al., 2000), and easier removal of the $\mathrm{Mg}^{2+}$ block of NMDA receptor attributable to membrane depolarization (Volgushev et al., 2000a,b). All of these factors are expected to lower the threshold for induction of plastic changes.

In addition, our EM results show that, at room temperature, in contrast to physiological temperature, even a relatively strong stimulus ( $10 \mathrm{~Hz}$ for $1 \mathrm{~min}$ ) mobilizes only a very small number of vesicles at a significant proportion of synapses (less than five vesicles at $\sim 5 \%$ of synapses). Such a small amount of release may be virtually undetectable with other methods, and these synapses may thus be classified as "presynaptically silent" (Kullmann, 2003; Voronin and Cherubini, 2003). Presynaptically silent synapses have been speculated to have an important role in synaptic plasticity as a "golden reserve" (Voronin and Cherubini, 2003). Our present results, however, caution that presynaptic silence could be an artifact of lowered experimental temperature.

Overall, our results strongly imply that synaptic plasticity induced at subnormal temperatures may not only be more pronounced but may also involve mechanisms that are not relevant at physiological temperatures. Bearing in mind that numerous other important aspects of synaptic function are also substantially influenced by temperature, it seems appropriate to propose that conclusions about physiological synaptic plasticity should only be drawn from studies at physiological temperatures.

\section{References}

Aravanis AM, Pyle JL, Tsien RW (2003) Single synaptic vesicles fusing transiently and successively without loss of identity. Nature 423:643-647. 
Betz WJ, Mao F, Bewick GS (1992) Activity-dependent fluorescent staining and destaining of living motor nerve terminals. J Neurosci 12:363-375.

Borst JG, Sakmann B (1998) Calcium current during a single action potential in a large presynaptic terminal of the rat brainstem. J Physiol (Lond) 506:143-157.

Cochilla AJ, Angleson JK, Betz WJ (1999) Monitoring secretory membrane with FM1-43 fluorescence. Annu Rev Neurosci 22:1-10.

David G, Barrett EF (2000) Stimulation-evoked increases in cytosolic $\left[\mathrm{Ca}^{2+}\right]$ in mouse motor nerve terminals are limited by mitochondrial uptake and are temperature dependent. J Neurosci 20:7290-7296.

De Camilli P, Slepnev VI, Shupliakov O, Brodin L (2001) Synaptic vesicle endocytosis. In: Synapses (Cowan MW, Südhof TC, Stevens CF, eds), pp 217-274. Baltimore: The Johns Hopkins University.

Dinkelacker V, Voets T, Neher E, Moser T (2000) The readily releasable pool of vesicles in chromaffin cells is replenished in a temperaturedependent manner and transiently overfills at $37^{\circ} \mathrm{C}$. J Neurosci 20:8377-8383.

Fernandez-Alfonso T, Ryan TA (2004) The kinetics of synaptic vesicle pool depletion at CNS synaptic terminals. Neuron 41:943-953.

Gandhi SP, Stevens CF (2003) Three modes of synaptic vesicular recycling revealed by single-vesicle imaging. Nature 423:607-613.

Goslin K, Asmussen H, Banker G (1998) Rat hippocampal neurons in lowdensity culture. In: Culturing nerve cells, Ed 2 (Banker G, Goslin K, eds), pp 339-370. Cambridge, MA: MIT.

Guibault GG (1990) General aspects of luminescence spectroscopy. In: Practical fluorescence (Guibault GG, ed), pp 1-40. New York: Marcel Dekker.

Harata N, Ryan TA, Smith SJ, Buchanan J, Tsien RW (2001a) Visualizing recycling synaptic vesicles in hippocampal neurons by FM 1-43 photoconversion. Proc Natl Acad Sci USA 98:12748-12753.

Harata N, Pyle JL, Aravanis AM, Mozhayeva M, Kavalali ET, Tsien RW (2001b) Limited number of recycling vesicles in small CNS nerve terminals: implications for neural signaling and vesicular cycling. Trends Neurosci 24:637-643.

Harris KM, Sultan P (1995) Variation in the number, location and size of synaptic vesicles provides an anatomical basis for the nonuniform probability of release at hippocampal CA1 synapses. Neuropharmacology 34:1387-1395.

Henkel AW, Lubke J, Betz WJ (1996) FM1-43 dye ultrastructural localization in and release from frog motor nerve terminals. Proc Natl Acad Sci USA 93:1918-1923.

Kirov SA, Petrak LJ, Fiala JC, Harris KM (2004) Dendritic spines disappear with chilling but proliferate excessively upon rewarming of mature hippocampus. Neuroscience 127:69-80.

Kullmann DM (2003) Silent synapses: what are they telling us about longterm potentiation? Philos Trans R Soc Lond B Biol Sci 358:727-733.

Li Z, Murthy VN (2001) Visualizing postendocytic traffic of synaptic vesicles at hippocampal synapses. Neuron 31:593-605.

Lisman J (2003) Long-term potentiation: outstanding questions and attempted synthesis. Philos Trans R Soc Lond B Biol Sci 358:829-842.

Lynch MA (2004) Long-term potentiation and memory. Physiol Rev 84:87-136.

Malenka RC, Siegelbaum SA (2001) Synaptic plasticity: diverse targets and mechanisms for regulating synaptic efficacy. In: Synapses (Cowan WM, Sudhof TC, Stevens CF, eds), pp 393-453. Baltimore: The Johns Hopkins University.

Mamdouh Z, Giocondi M-C, Laprade R, Grimellec CL (1996) Temperature dependence of endocytosis in renal epithelial cells in culture. Biochim Biophys Acta 1282:171-173.

McNaughton BL, Shen J, Rao G, Foster TC, Barnes CA (1994) Persistent increase of hippocampal presynaptic axon excitability after repetitive electrical stimulation: dependence on $N$-methyl-D-aspartate receptor activity, nitric-oxide synthase, and temperature. Proc Natl Acad Sci USA 91:4830-4834.
Micheva KD, Buchanan J, Holz RW, Smith SJ (2003) Retrograde regulation of synaptic vesicle endocytosis and recycling. Nat Neurosci 6:925-932.

Murthy VN, Stevens CF (1999) Reversal of synaptic vesicle docking at central synapses. Nat Neurosci 2:503-507.

Neale EA, Bowers LM, Jia M, Bateman KE, Williamson LC (1999) Botulinum neurotoxin A blocks synaptic vesicle exocytosis but not endocytosis at the nerve terminal. J Cell Biol 147:1249-1260.

Pyle JL, Kavalali ET, Piedras-Renteria ES, Tsien RW (2000) Rapid reuse of readily releasable pool vesicles at hippocampal synapses. Neuron 28:221-231.

Pyott SJ, Rosenmund C (2002) The effects of temperature on vesicular supply and release in autaptic cultures of rat and mouse hippocampal neurons. J Physiol (Lond) 539:523-535.

Regehr WG, Stevens CF (2001) Physiology of synaptic transmission and short-term plasticity. In: Synapses (Cowan WM, Südhof TC, Stevens CF, eds), pp 135-175. Baltimore: The Johns Hopkins University.

Rizzoli SO, Betz WJ (2004) The structural organization of the readily releasable pool of synaptic vesicles. Science 303:2037-2039.

Roelandse M, Matus A (2004) Hypothermia-associated loss of dendritic spines. J Neurosci 24:7843-7847.

Ryan TA (2001) Presynaptic imaging techniques. Curr Opin Neurobiol 11:544-549.

Ryan TA, Smith SJ (1995) Vesicle pool mobilization during action potential firing at hippocampal synapses. Neuron 14:983-989.

Ryan TA, Ziv NE, Smith SJ (1996) Potentiation of evoked vesicle turnover at individually resolved synaptic boutons. Neuron 17:125-134.

Ryan TA, Reuter H, Smith SJ (1997) Optical detection of a quantal presynaptic membrane turnover. Nature 3888:478-482.

Schikorski T, Stevens CF (1997) Quantitative ultrastructural analysis of hippocampal excitatory synapses. J Neurosci 17:5858-5867.

Shen K-F, Schwartzkroin PA (1988) Effects of temperature alterations on population and cellular activities in hippocampal slices from mature and immature rabbit. Brain Res 475:305-316.

Silva AJ (2003) Molecular and cellular cognitive studies of the role of synaptic plasticity in memory. J Neurobiol 54:224-237.

Südhof TC (2004) The synaptic vesicle cycle. Annu Rev Neurosci 27:509547.

Thompson SM, Masukawa LM, Prince DA (1985) Temperature dependence of intrinsic membrane properties and synaptic potentials in hippocampal CA1 neurons in vitro. J Neurosci 5:817-824.

Volgushev M, Vidyasagar TR, Chistiakova M, Eysel UT (2000a) Synaptic transmission in the neocortex during reversible cooling. Neuroscience 98:9-22.

Volgushev M, Vidyasagar TR, Chistiakova M, Yousef T, Eysel UT (2000b) Membrane properties and spike generation in rat visual cortical cells during reversible cooling. J Physiol (Lond) 522:59-76.

Volgushev M, Kudryashov I, Chistiakova M, Mukovski M, Niesmann J, Eysel UT (2004) Probability of transmitter release at neocortical synapses at different temperatures. J Neurophysiol 92:212-220.

Voronin LL, Cherubini E (2003) "Presynaptic silence" may be golden. Neuropharmacology 45:439-449.

Xia Z, Dudek H, Miranti CK, Greenberg ME (1996) Calcium influx via the NMDA receptor induces immediate early gene transcription by a MAP kinase/ ERK-dependent mechanism. J Neurosci 16:5425-5436.

Zakharenko SS, Zablow L, Siegelbaum SA (2001) Visualization of changes in presynaptic function during long-term synaptic plasticity. Nat Neurosci 4:711-717.

Zakharenko SS, Zablow L, Siegelbaum SA (2002) Altered presynaptic vesicle release and cycling during mGluR-dependent LTD. Neuron 35:1099-1110.

Zenisek D, Steyer JA, Feldman ME, Almers W (2002) A membrane marker leaves synaptic vesicles in milliseconds after exocytosis in retinal bipolar cells. Neuron 35:1085-1097. 